\title{
Interview with Karim Adiprasito
}

Toufik Mansour

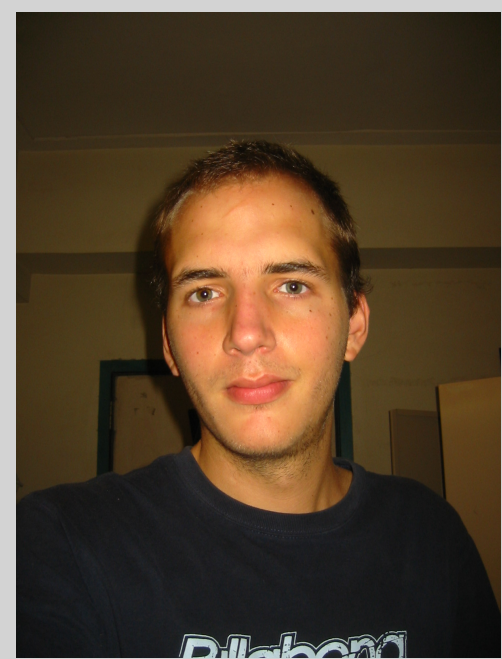

Karim Adiprasito completed his undergraduate studies at TU Dortmund in 2010. He obtained a Ph.D. from Free University Berlin in 2013, under the supervision of Günter M. Ziegler. He has been an Assistant Professor at the Einstein Institute for Mathematics Hebrew University of Jerusalem since 2015, promoted to full professor in 2018. Professor Adiprasito has given numerous invited talks in conferences and seminars. He received several prizes, for example, European Prize in Combinatorics, in 2015, for his work in discrete geometry; New Horizons Prize for Early-Career Achievement in Mathematics, associated with the Breakthrough Prize in Mathematics, in 2019; Prize of the European Mathematical Society in 2020, and is the 2021 Hadamard Lecturer.
Mansour: Professor Adiprasito, first of all, we would like to thank you for accepting this interview. Would you tell us broadly what combinatorics is?

Adiprasito: In my perspective, combinatorics is a common ground in mathematics. It is a place where everything comes together, which has limitless applications to other fields, as well as being the playground for other fields. What is most fascinating to me though is its ubiquity in everything in daily life, as our lives are naturally discrete. As such, when we speak of a continuous process, we often mean a really large discrete one, and so the discrete and the singular is really the germane issue to look at when we want to find out the limits of our reasoning.

Combinatorics is also just how we like to think, how we like to order things in our minds. It was always fascinating to me that the ancient Greeks chose polyhedra to model nature, pointy and unwieldy shapes, instead of the circle.

Mansour: What do you think about the development of the relations between combina- torics and the rest of mathematics?

Adiprasito: I am not a fan of isolated combinatorics as a field or any field for that matter. It is to be primarily a place to meet and discuss mathematical problems from various areas. I do not feel I am particularly talented in mathematics overall, so translating a problem to a combinatorial one is always helpful to me to grasp it for myself, and then hopefully to solve it.

Mansour: What have been some of the main goals of your research?

Adiprasito: Good question (in the sense that I needed to think about it for a bit). I was always more guided by cool ideas rather than goals, and I am rather impulsive at that. So if I find some interesting idea, have an interesting thought, then I would just try it out. But I am not someone that sits and broods over a specific problem for a long time unless he sees a crack in the wall. I have a collection of problems in the back of my head, but I consider this collection more as a playground to test ideas on.

Mansour: We would like to ask you about

The authors: Released under the CC BY-ND license (International 4.0), Published: June 18, 2021

Toufik Mansour is a professor of mathematics at the University of Haifa, Israel. His email address is tmansour@univ.haifa.ac.il 
your formative years. What were your early experiences with mathematics? Did that happen under the influence of your family or some other people?

Adiprasito: I think the earliest encounter with mathematics is from riddles my grandfather asked me. I must say, I never liked riddles. It always feels annoying to be put on the spot, and be asked to come up with an idea right there and then. It always feels more like a test than an invitation to think.

So I think the first time I actually liked mathematics was when I discovered it myself. One instance from school I remember vividly was not actually in a mathematics class (which I found to be mostly average and boring, especially towards the final years. The teachers were just not competent) but from social science class (or rather, the first course in economics) where I learned, though in a hidden way, about the connection between economic (or rather, game-theoretic) equilibria and fixed point theorems.

Mansour: Were there specific problems that made you first interested in combinatorics?

Adiprasito: I think the first encounter with combinatorics that made me go "huh" was reading about spaces of bounded curvature, in relation to hyperbolic groups and Alexandrov's curvature notions ${ }^{1}$. That does not seem combinatorial at first, but the remarkable thing was that Gromov ${ }^{2}$ really turned a geometric property into a combinatorial one, what he called the no-triangle condition (graph theorists would call it the clique complex). That stuck with me for a long time, because it was in geometry textbooks and contexts that I really encountered the first (specifically, Gromov's textbook ${ }^{3}$ on Metric Structures on Riemannian and Non-Riemannian spaces). I noticed for myself that Gromov was really being a combinatorialist at heart when he wrote this, and I found the connection immensely impressive.
Also, it has remained as my favorite book.

Mansour: What was the reason you chose the Free University Berlin for your Ph.D. and your advisor Günter M. Ziegler?

Adiprasito: I was honestly supposed to go to the United States for graduate school (I had several offers, but ultimately hoped to work with Gromov at Courant). But, for personal reasons, I decided to stay in Germany, so I contacted Guenter M. Ziegler while I was vacationing in Berlin. We talked about some work we did, and to be honest, I think I selected him out of personal compatibility just as much as I was interested in polytopes. He let me do research mostly on my own terms, but we had some nice projects together as well.

Mansour: What was the problem you worked on in your thesis?

Adiprasito: Several, actually. First, there was a postdoc present there in my first year, Bruno Benedetti, with whom I met out of principle in some Italian restaurant in Berlin instead of the office. This was quite convenient, as I was honestly not a good office student, and never became one. Anyway, we worked on various questions in geometric topology, mostly related to the question of how difficult geometric triangulations can be. One cute fact that came out of that was that at some point I realized how to prove ${ }^{4}$ the Hirsch conjecture ${ }^{5}$ for flag complexes using Gromov's work.

And then I studied a problem concerning arrangement theory that had been looked at by Salvetti before, describing complements of arrangements. Essentially, I wanted to show that they are minimal in a homotopic sense. I realized that there was a simple way to do it via combinatorial trick and in particular proved ${ }^{6}$ the minimality of some arrangement complements.

And then finally, the "magnum opus" was something that came out of a discussion with Günter Ziegler, where we studied a very old

\footnotetext{
1 A. D. Alexandrov, V. N. Berestovskii and I. G. Nikolaev, Generalized Riemannian spaces, Russian Math. Surveys 41 (1986), $1-54$.

${ }^{2}$ M. Gromov, Hyperbolic groups, In S. Gersten, editor, Essays in Group Theory, Volume 8 of Mathematical Sciences Research Institute Publications, 75-263, Springer, New York, 1987.

${ }^{3}$ M. Gromov, Metric structures for Riemannian and non-Riemannian spaces, Progress in Mathematics 152 , Birkhäuser, Boston, 1999.

${ }^{4}$ K. A. Adiprasito and B. Benedetti, The Hirsch conjecture holds for normal flag complexes, Mathematics of Operations Research 39:4 (2014), 1340-1348.

${ }^{5}$ G. B. Dantzig, Linear Programming and Extensions, Princeton University Press, Princeton, N.J., 1963.

${ }^{6}$ K. A. Adiprasito, Combinatorial stratifications and minimality of 2-arrangements, J. Topology 7:4 (2014), 1200-1220.

${ }^{7}$ E. Steinitz, Polyeder und Raumeinteilungen, Encyklopädie der mathematischen Wissenschaften, Dritter Band: Geometrie, III.1.2., Heft 9, Kapitel III A B 12 (W. Fr. Meyer and H. Mohrmann, eds.), B. G. Teubner, Leipzig, 1922, 1-139. xi, 53.
} 
conjecture of Legendre and Steinitz ${ }^{7}$ on the dimension of deformation space of polytopes. The construction is a "Berlin" one, in the sense that we realized that certain discretizations of partial differential equations (used for instance in architecture) could be used to solve the problem for us.

Mansour: What would guide you in your research?, a general theoretical question or a specific problem?

Adiprasito: I mostly said this already: neither. It is mostly a neat idea that I obsess over, and it can be motivated by either general or specific problems. Having understood something about the inner workings though is what is leads my research.

Mansour: When you are working on a problem, do you feel that something is true even before you have the proof?

Adiprasito: Yes, but that feeling can be treacherous. Intuition tends to misguide me on occasion, but in general, it is a rather good companion. I had cases where something that intuitively should have been one way turned out to be another, and these are usually the most interesting cases.

Mansour: What three results do you consider the most influential in combinatorics during the last thirty years?

Adiprasito: Probably, in no particular order (and I should say they are selected for their implications and the work they spawned more than the individual results):

1. Peter McMullen's ${ }^{8}$ work on valuation and polytope algebras, which opened up a (convex)geometric avenue to algebraic geometry. It is quite an ingenious perspective, which in particular also leads to our solution ${ }^{9}$ of Rota's conjecture and the Elias-Williamson work on the positivity of Kazhdan-Lusztig polynomials.

2. Gromov's ${ }^{10}$ geometric measure theory approach to expansion and multi-covered points that ingeniously connected several mea- sures of complexity for hypergraphs. It is one aspect of the story of high-dimensional expansion that I find very fascinating, and it masterfully uses rather difficult geometric arguments to achieve it.

3. I very much love Sabitov's proof ${ }^{11}$ of Bellow's conjecture, that is, that the volume of a flexible polyhedron is constant. It is a very beautiful result connecting geometry, algebra, and combinatorics, and several questions remain open. It is elegant and uses several interesting ideas masterfully.

Mansour: What are the top three open questions in your list?

Adiprasito: I am very much interested at the moment in the Hopf conjecture, that describes the Euler characteristic of aspherical manifolds. It is very much connected to combinatorics and group theory with algebraic geometry, in very mysterious and poorly understood ways. Specifically ${ }^{12}$, it says that the Euler characteristic $\chi(M)$ of a $2 n$-dimensional compact closed manifold satisfies

$$
(-1)^{n} \chi(M) \geq 0 .
$$

I also think that several questions in graph theory need to be understood (better), such as the strong perfect graph theorem ${ }^{13}$ or the graph reconstruction conjecture ${ }^{14}$ (which asks if a graph can be reconstructed if we only see all the versions with one edge removed.) Another reconstruction conjecture, whether a simple cellulation of a sphere can be reconstructed from its 1-skeleton, is equally fascinating and shows how little we know about graph embeddings in higher dimensions.

And finally, I am interested in the problem of renormalization in general relativity, and models of quantum gravity. I only recently got interested in mathematical physics, but the amount of mathematics that comes together in this topic is fascinating. It is also related to combinatorial problems, such as the prob-

\footnotetext{
${ }^{8}$ P. McMullen, The polytope algebra, Adv. Math. 78 (1989), 76-130

${ }^{9}$ K. Adiprasito, J. Huh, and E. Katz, Hodge theory for combinatorial geometries, Ann. of Math. (2) 188:2 (2018), 381-452.

${ }^{10}$ M. Gromov, Singularities, expanders, and topology of maps. Part 2: From combinatorics to topology via algebraic isoperimetry, Geom. Funct. Anal. 20(2) (2010), 416-526.

${ }^{11}$ I. Kh. Sabitov, The volume as a metric invariant of polyhedra, Discrete Comput. Geom. 20 (1998), $405-425$.

${ }^{12}$ See, for example, J. Cao and F. Xavier, Kähler parabolicity and the Euler number of compact manifolds of non-positive sectional curvature, Math. of Ann. 319 (2001), 483-491.

${ }^{13}$ M. Chudnovsky, Maria, N. Robertson, P. Seymour, and R. Thomas, The strong perfect graph theorem, Ann. of Math. 164 (1) (2006), 51-229.

${ }^{14} \mathrm{~F}$. Harary, On the reconstruction of a graph from a collection of subgraphs, In Theory of Graphs and its Applications (Proc. Sympos. Smolenice, 1963). Publ. House Czechoslovak Acad. Sci., Prague, 1964, 47-52.

${ }^{15}$ See, for example, https://arxiv.org/pdf/1904.01966.pdf.
} 
lem of counting the number of triangulations of manifolds (so-called Regge calculus ${ }^{15}$ ).

Mansour: What kind of mathematics would you like to see in the next ten-to-twenty years as a continuation of your work?

Adiprasito: I think one thing that I would love to see is a deeper understanding of differential geometry at the singular limit. In particular, there are invariants of manifolds that we know only to compute if we have a triangulation or a smooth structure, but no unifying theory of both. So geometric and combinatorial questions still feel kind of disjoint. In particular, many things are proven twice in very similar forms, once for combinatorics and once in geometry, which feels a little strange at times.

Mansour: Do you think that there are core or mainstream areas in mathematics? Are some topics more important than others?

Adiprasito: I do not think so. On the contrary, I think it rather frustrating if researchers stay firmly rooted within their area, as it often prevents them from seeing the bigger picture. Still, to stay in their special corner is something mathematicians of many areas do, unfortunately.

Mansour: What do you think about the distinction between pure and applied mathematics that some people focus on? Is it meaningful at all in your case? How do you see the relationship between so-called "pure" and "applied" mathematics?

Adiprasito: I think the distinction is more one of "we stop once we have the solution to the technical problem posed" and "we want to understand the bigger picture." I think the former is certainly less exciting to me, but it is less a distinction of pure vs. applied than an unwillingness to go on beyond what you were asked.

Mansour: What advice would you give to young people thinking about pursuing a research career in mathematics?

Adiprasito: I think the best advice is to not confine yourself to a single area, and not be afraid to understand ideas regardless of where they are coming from. It gives a better scope of what you are doing and helps you understand the relevance of your research. And of course, it gives you more tools at your disposal.

Mansour: Would you tell us about your in- terests besides mathematics?

Adiprasito: I like literature, both in the sense that I write prose myself and read constantly. Whenever I do not read math, I usually have a novel in my hand. Mostly though, I love cooking. It is the mix of craft and creativity that is only found again in mathematics, as it is both an art and bound to real laws (after all, you do not want to poison everyone at the table.)

Mansour: This is a quote by John Fraleigh that says "Never underestimate a theorem that counts something." How far you agree with it? To what extent do you agree with this quote? Adiprasito: I never was a good quotes person, honestly. It mostly feels as if they should have stayed in their own time, as part of their conversation, their context. The closest thing I like is, perhaps, poetry. And I think I like Rilke best most of the time. The Panther in particular has stayed with me for a long time. Mansour: Recently, Professor Abigail Thompson, a Vice President of the AMS, expressed her view on a mandatory "Diversity Statement" for job applicants in a short essay. Apparently, and unfortunately, there were several attempts from members of the mathematical community to intimidate her 'voice'. You were among the undersigned mathematician urging the AMS 'to stand by the principle that important issues should be openly discussed respectfully...'. Was your petition helpful? When should the issue of diversity be taken into consideration? How negatively can such a mandatory act impact future research? Adiprasito: I think I was mostly taken aback by the tone of the discussion. That said, I do not believe forcing faculty to write a diversity statement is useful, and it is the lazier and the cheaper way of universities to feign activity in this issue. A more helpful measure would be to make life easier for young families by providing childcare, parental leave, or to improve conditions of students that do not have adequate financial means.

Mansour: You recently started a blog. You describe it as "A blog on mathematical musings, nonsense, and observations that did not make it into a paper." In what sense do you use the word 'nonsense'? How much time do you plan to dedicate to it?

Adiprasito: Well, I do expect that I might also share ideas that do not work out or ran- 
dom intuitions that might be wrong. In that sense, nonsense. Mostly though, nonsense pertained to little observations I had, that I felt would not be worth a paper but that should still be recorded somewhere. I do not know how much time will I dedicate to it.

Mansour: Recently you were awarded the prestigious New Horizons Prize in Mathematics for the development of combinatorial Hodge theory leading to the resolution of the log-concavity conjecture of Heron-Rota-Welsh. What is Hodge theory? Especially, in joint work with June Huh and Eric Katz ${ }^{9}$, you resolved the Heron-Rota-Welsh conjecture on the log-concavity of the characteristic polynomial of matroids. Would you tell us about this result, the crucial ideas behind the proof, and the possible future research directions?

Adiprasito: Hodge Theory is a way to study the cohomology of manifolds and varieties, but in this context, it should be taken more as a way to study the signature of certain bilinear forms associated with matroids. This is really a special part of Hodge theory though. The combinatorics mostly enters when proving that the bilinear forms indeed say something about the coefficients of the characteristic polynomial.

The idea is quite simple: log-concavity of sequences $a_{i}$ can be restated as saying that a certain matrix, the matrix

$$
\left(\begin{array}{cc}
a_{i+1} & a_{i} \\
a_{i} & a_{i-1}
\end{array}\right)
$$

has non-positive determinant, or equivalently, it cannot be definite. To prove that, one needs to establish that the matrix arises as a bilinear form that has a geometric meaning, in our case, the Hodge-Riemann relations. Proving them is the major feat of our joint work, as we had to reprove a classical algebraic geometry result in a much larger generality than previously known. The limits of the latter are the most interesting to me and remain to be explored.

Mansour: Another great piece of your work, carried out together with Bruno Benedetti ${ }^{4}$, is the resolution of the Hirsch conjecture for flag triangulations of manifolds. Would you elab- orate on the result by emphasizing a little bit more on the combinatorial side of it?

Adiprasito: Well, the Hirsch conjecture, in a more general setting, asks whether the diameter of the hypergraph of facets of a triangulated manifold is bounded, say polynomially, in terms of the number of vertices. This is a rather intriguing conjecture, but so far, poorly understood. What I observed is that for hypergraphs arising as clique complexes (this is also known as the no-triangle condition), one could use a method developed by Gromov to prove the strongest form of this conjecture. Essentially, one constructs the shortest path on vertices geometrically, and from this, one is able to construct a short path in the hypergraph.

Mansour: You were awarded the European Mathematical Society prize for your work ${ }^{16}$, Peter McMullen's g-conjecture for simplicial spheres. We would like to hear your comments about the proof. What do you think about the role of prizes such as this one in mathematical research?

Adiprasito: This is a far-reaching generalization of the work of McMullen, as well my joint work with June and Eric on the Rota conjecture. Essentially, when in the case of matroids, we had a geometric framework to work with, specifically a notion of convexity, played by submodular functions, the $g$-conjecture had to come from a direction entirely without geometry. I managed to do that by connecting the problem to an algebraic version of Hall's marriage theorem, which yielded the desired after much additional work. In particular, I noticed that what was needed is the fact that in a Poincaré duality algebra associated with the problem, the face ring, the pairing needs to stay non-degenerate when restricted to ideals. This property, called biased pairing, as central as it turned out. Recently, with Papadakis and Petrotou ${ }^{17}$ we gave another proof based on perturbation of volume polynomials which seems more miraculous and remains to be understood, but that again uses the biased pairing property critically.

Mansour: In a very recent paper, you and Raman Sanyal ${ }^{18}$, have solved two long-standing problems on the combinatorial complexity of

\footnotetext{
${ }^{16}$ K. Adiprasito, Combinatorial Lefschetz theorems beyond positivity, arXiv:1812.10454.

${ }^{17}$ See https : //arxiv.org/pdf/2101.07245.pdf.

${ }^{18}$ K. A. Adiprasito and R. Sanyal, Relative Stanley-Reisner theory and upper bound theorems for Minkowski sums, Publications mathématiques de l'IHÉS, (2016), 1-65.
} 
Minkowski sums of polytopes. Would you tell us about this work and point out some possible future research directions?

Adiprasito: The problem is rather simple to state: given polytopes, how complex can their Minkowski sum be, and how complex does it have to be. For instance, we could fix the number of vertices of each summand and could ask for the number of vertices in the Minkowski sum. That problem sounds rather innocent, but solving it requires a rather powerful geometric construction, the Cayley polytope, plus a lot of commutative algebra and combinatorics, to relate the complexity of summands to the complexity of the sum. But of course, one can ask many other questions concerning the complexity of Minkowski sums. In general, it is a broader problem of interest to understand how combinatorial objects mix, and I feel that often algebraic methods are the right way to understand it.

Mansour: You have worked on outstanding conjectures and solved some of them. What is your initial approach towards conjectures? Would you describe some of your experiences? Adiprasito: I usually try to get a feeling for conjectures by connecting to my knowledge of techniques and intuitions from different areas of mathematics. That usually gets me to a point where I feel I understand the conjecture well enough to at least be able to say something relevant. However, that is just where the process starts, and it takes a lot of work after that.

Mansour: In your work, you have extensively used combinatorial reasoning to address important problems. How do enumerative techniques engage in your research?

Adiprasito: Actually, I often do not use so much combinatorial reasoning, but reasoning from various areas. Indeed, the contrary often helps me, as I try to imagine how a combina- torial problem would look if looked at continuously, and then try to see where the boundary in the behavior is.

Mansour: Would you tell us about your thought process for the proof of one of your favorite results? How did you become interested in that problem? How long did it take you to figure out a proof? Did you have a "eureka moment"?

Adiprasito: I usually do have several eureka moments, few of which actually work out in the end. My proof of $g$-conjecture is a bad example probably, as several were needed to complete the proof. I think I needed a year to work out all the steps. On the other hand, in the case of the Rota conjecture, once the idea was there, it took like a week to have everything together in my head.

I think I most vividly remember filling in a specific step in the proof of the $g$-conjecture though when I realized that a lemma of Kronecker I used works together beautifully with Poincaré duality algebras (you can find the more technical details in my recent newsletter of the EMS survey). I was walking home from a meeting with my friend and colleague Eran Nevo, and decided to have some wonderful ice cream instead, and realized the connection when enjoying some saffron ice.

Mansour: Is there a specific problem you have been working on for many years? What progress have you made?

Adiprasito: I worked for a long time on the $g$-conjecture, but currently I do not have a big problem that occupies me constantly. It is more the case that I am trying to understand some fundamental issues in graph theory a bit better.

Mansour: Professor Karim Adiprasito, I would like to thank you for this very interesting interview on behalf of the journal Enumerative Combinatorics and Applications. 